OPEN ACCESS

Edited by: Janine Lamb,

The University of Manchester, United Kingdom

Reviewed by: Banu Peynircioğ/u, Hacettepe University, Turkey

Hui Li,

Guangxi University, China Joanna Parkes, Binghamton University, United States

*Correspondence: Guochun Wang guochunwang@hotmail.com Xiaoming Shu

sxm992283@hotmail.com

${ }^{\dagger}$ These authors have contributed equally to this work

Specialty section: This article was submitted to Autoimmune and Autoinflammatory Disorders, a section of the journal

Frontiers in Immunology

Received: 20 September 2021 Accepted: 13 December 2021 Published: 05 January 2022

Citation:

Ye L, Zuo Y, Chen F, Peng Q, LuX, Wang G and Shu X (2022) miR-18a-3p and lts Target Protein HuR May Regulate Myogenic

Differentiation in ImmuneMediated Necrotizing Myopathy.

Front. Immunol. 12:780237. doi: 10.3389/fimmu.2021.780237

\section{miR-18a-3p and Its Target Protein HuR May Regulate Myogenic Differentiation in Immune-Mediated Necrotizing Myopathy}

\author{
Lifang Ye ${ }^{1,2 t}$, Yu Zuo ${ }^{1 \dagger}$, Fang Chen ${ }^{1}$, Qinglin Peng ${ }^{1}$, Xin Lu ${ }^{1}$, Guochun Wang ${ }^{1,2 *}$ \\ and Xiaoming Shu ${ }^{1 *}$
}

\footnotetext{
${ }^{1}$ Department of Rheumatology, Key Laboratory of Myositis, China-Japan Friendship Hospital, Beijing, China, ${ }^{2}$ Peking Union Medical College, Chinese Academy of Medical Sciences, Beijing, China
}

Immune-mediated necrotizing myopathy (IMNM) is characterized by manifestation of myonecrosis and regeneration of muscle fibers; however, the underlying pathogenesis remains unclear. This study aimed to investigate the role and mechanism of miR-18a-3p and its target RNA-binding protein HuR in IMNM. HuR and miR-18a-3p levels were detected in the skeletal muscles of 18 patients with IMNM using quantitative reversetranscription real-time polymerase chain reaction (qRT-PCR) and western blotting analysis. Human myoblasts were transfected with small interfering RNA targeting HuR and miR-18a-3p mimic or inhibitor. Myogenic differentiation markers, myogenin and myosin heavy chain, were analyzed by qRT-PCR, western blotting analysis, and immunofluorescence staining. The results showed that miR-18a-3p was upregulated $(p=0.0002)$, whereas HuR was downregulated $(p=0.002)$ in the skeletal muscles of patients with IMNM. The expression of miR-18a-3p in patients with IMNM was negatively correlated with those of $\operatorname{HuR}(r=-0.512, p=0.029)$. We also found that disease activity was positively correlated with HuR expression ( $r=0.576, p=0.012)$ but muscle activity was negatively correlated with miR-18a-3p expression $(r=-0.550, p=$ 0.017). Besides, bioinformatics analysis and dual-luciferase reporter assays suggested that miR-18a-3p could directly target HuR. Cellular experiments showed that overexpression of miR-18a-3p inhibited myogenic differentiation by targeting HuR, whereas inhibition of miR-18a-3p led to opposite results. Therefore, miR-18a-3p and its target protein HuR may be responsible for modulating the myogenic process in IMNM and can thus be therapeutic targets for the same.

Keywords: HuR, miR-18a-3p, RNA binding protein, immune-mediated necrotizing myopathy, myogenic differentiation

\footnotetext{
Abbreviations: IMNM, immune-mediated necrotizing myopathy; UTR, untranslated region; HSMM, human skeletal muscle myoblasts; ELISA, enzyme-linked immunosorbent assay; PGA, physician global assessment; MMT8, manual muscle testing 8; HC, healthy controls; MyoG, myogenin; IIM, idiopathic inflammatory myopathy; RBP, RNA-binding protein; HMGCR, anti3-hydroxy-3-methylglutaryl-CoA reductase; anti-SRP, anti-signal recognition particle; qRT-PCR, quantitative reversetranscription real-time polymerase chain reaction.
} 


\section{INTRODUCTION}

Immune-mediated necrotizing myopathy (IMNM) is an autoimmune disease that is characterized by severe muscle weakness and myofiber necrosis, but usually low inflammatory cell infiltration and lesser involvement of other organs (1). IMNM consists of three subclasses: anti-signal recognition particle (anti-SRP)-positive, anti-3-hydroxy-3-methylglutarylCoA reductase (HMGCR)-positive, and seronegative (2). Myoblast fusion in patients with IMNM is abnormal since the mechanism involved in muscle regeneration is impaired (3); however, the pathogenesis of IMNM is still unclear.

MicroRNAs (miRNA) participate in skeletal muscle differentiation (4). miR-17-92, a highly conserved miRNA cluster, comprises miR-17, miR-20a, miR-18a, miR-19a, miR19b-1, and miR-92a-1; these miRNAs play an essential role in cell proliferation, differentiation, tumorigenesis, and angiogenesis (5-7). The pathogenic role of miRNAs in many diseases, including autoimmune diseases, has been extensively studied. Previous studies have shown that miRNA expression is abnormal in the peripheral blood mononuclear cells, skeletal muscles, plasma, serum, and lungs of patients with idiopathic inflammatory myopathy (IIM); thus, dysregulated miRNA expression may be involved in disease pathogenesis (8-11). Besides, the expression of inflammatory miRNAs and antitroponin-targeted miRNAs is increased in mice with severe myositis, indicating that miRNAs are involved in muscle injury, inflammation, and myasthenia (12). Our previous study also revealed that several immune-related miRNAs are correlated with dermatomyositis (13); however, the involvement of miRNAs in IMNM remains unclear.

RNA-binding proteins (RBPs) are known to change the fate or function of bound RNA. Of such RBPs, HuR, a member of the ELAVL1/Hu family, has been reported to modulate the fusion of myoblasts into myotubes during skeletal myogenesis by coordinating the expression of myogenic regulatory factors such as myogenin (MyoG), MyoD, and p21 (14-16). Nevertheless, the role of HuR in IMNM has not been investigated.

In this study, we aimed to explore the role and mechanism of abnormally expressed miR-18a-3p and its target RBP HuR in the myogenesis of patients with IMNM. Our results may help in better understanding the pathogenesis of IMNM and developing effective therapeutic targets.

\section{MATERIALS AND METHODS}

\section{Study Population}

Patients with IMNM, DM and healthy controls (HCs), who participated in this study, were admitted to the Department of Rheumatology and Physical Examination from 2017 to 2019 in China-Japan Friendship Hospital, Beijing, China. The age and gender of the patients with IMNM were matched with those of the HCs. IMNM was classified according to the pathological criteria described at the 224th European Neuromuscular Center International Workshop (17). This study did not include patients with other autoimmune diseases, tumors, and infections. Skeletal muscle tissue biopsies were obtained from 18 patients with IMNM, 11 patients with DM and 8 HCs. The study was approved by the Ethical Review Committee of the ChinaJapan Friendship Hospital (2019-25-K19). Before commencing the study, we obtained written informed consent from all patients for the use of their tissues and data.

\section{Clinical Assessment}

Detailed results of physical examinations and routine laboratory tests of all patients were recorded at their first visit. Routine laboratory parameters, including the levels of serum C-reactive protein, creatine kinase, alanine aminotransferase, aspartate aminotransferase, and lactate dehydrogenase, and erythrocyte sedimentation rate, were assessed. The damage to the patients with IMNM was evaluated using manual muscle testing 8 (MMT8). A $10 \mathrm{~cm}$ visual analog scale (VAS) was used to evaluate the muscle activity component of the myositis disease activity assessment tool (MDAAT-muscle) and physician global assessment (PGA) for disease activity (18).

Serum anti-HMGCR antibodies were detected using an enzyme-linked immunosorbent assay (ELISA) kit (Inova Diagnostics, San Diego, CA, USA). Serum anti-SRP antibodies were detected using a commercially available kit (Euroimmun, Lübeck, Germany).

\section{Myoblast Culture and Differentiation}

Human skeletal muscle myoblasts (HSMM) were obtained from Lonza Bioscience (Basel, Switzerland) and cultured using the Clonetics skeletal muscle myoblast cell system (Lonza) in a humidified incubator (Thermo Fisher Scientific, Waltham, MA, USA) at $37^{\circ} \mathrm{C}$ with $5 \% \mathrm{CO}_{2}$. The cells were seeded in 12-well or 6 -well plates and incubated for $18 \mathrm{~h}$ before transfection. At 80 $90 \%$ confluence, the cells were transferred into Dulbecco's modified Eagle medium supplemented with $2 \%$ horse serum and $1 \%$ penicillin-streptomycin for inducing differentiation.

\section{Cell Transfection}

HSMM were seeded in 12-well or 6-well plates; when the cells reached approximately $70 \%$ confluence, they were transfected with $100 \mathrm{nM}$ of small interfering RNA targeting $\mathrm{HuR}$ (siHuR) or small interfering control RNA (siCtrl), miR-18a-3p mimics or mimics negative control (NC) and miR-18a-3p inhibitor or inhibitor NC (Ribobio, Guangzhou, China) using Lipofectamine 3000 (Invitrogen, Carlsbad, CA, USA) according to the manufacturer's instructions. The culture system was replaced with fresh medium at $12 \mathrm{~h}$ post-transfection. The cells were used to stimulate differentiation at $24 \mathrm{~h}$ post-transfection.

\section{Dual-Luciferase Reporter Gene Assay}

Dual-luciferase reporter gene assay was used to confirm the binding sites of miR-18a-3p and the $3^{\prime}$ untranslated region (UTR) of HuR mRNA. A pmirGLO-HuR-wild type (Hur-WT) or a pmirG-LO-HuR-mutant type (HuR-MUT) and miR-18a-3p 
mimics or mimics NC were co-transfected into HEK293T cells using Lipofectamine 2000 (Invitrogen). Dual-Glo luciferase assay system (Promega, Madison, WI, USA) was used to measure the luciferase activity in the cell lysates according to the manufacturer's instructions. The ratios of Firefly to Renilla luciferase activities were used as the result of the dualluciferase reporter gene assay. The experiments were performed in three independent replicates.

\section{Bioinformatics Analysis}

The online databases miRwalk (http://mirwalk.umm.uniheidelberg.de/) and TargetScan (http://www.targetscan.org/ vert_72/) were used to search for the targets of miR-18a-3p $(19,20)$. Venny (https://bioinfogp.cnb.csic.es/tools/venny/index. html) was used to obtain the common targets from above mentioned databases. Heatmap software(ggplot2) was applied to cluster the enrichment terms obtained after Kyoto Encyclopedia of Genes and Genomes-based analysis of the common genes.

\section{RNA Extraction and Quantitative Reverse- Transcription Polymerase Chain Reaction}

Trizol (Invitrogen) was used to extract total RNA from the muscle tissues according to the manufacturer's instructions. PrimeScript $^{\mathrm{TM}}$ RT reagent Kit (Takara, Otsu, Japan) was used to reverse transcribe RNA into complementary DNA (cDNA). Quantitative reverse-transcription PCR (qRT-PCR) was performed using an ABI 7500 sequence detection system (Applied Biosystems, Foster City, CA) with SYBR Green Master Mix (Qiagen, Hilden, Germany). The thermal conditions were as follows: $95^{\circ} \mathrm{C}$ for $2 \mathrm{~min}$, followed by 40 cycles at $95^{\circ} \mathrm{C}$ for $5 \mathrm{~s}$ and $60^{\circ} \mathrm{C}$ for $30 \mathrm{~s}$. GAPDH was used as the internal reference of gene expression. Each sample was measured in triplicate. The U6 small RNA was used as the internal reference for estimating the levels of miR-18a-3p and other members in miR-17-92 cluster. All relevant PCR primers used in this study are listed in Supplementary Table 1.

\section{Western Blotting Analysis}

NE-PER Nuclear and Cytoplasmic Extraction Reagents kit (Thermo Fisher Scientific) was used to extract nuclearcytoplasmic fraction according to the manufacturer's instructions. NP-40 lysis buffer (Beyotime, Shanghai, China) and phenylmethylsulfonyl fluoride (Solarbio, Beijing, China) were used to obtain total protein. Protein quantification were performed by BCA Protein Assay kit (Thermo Fisher Scientific) as per the manufacturer's instructions. The available protein was denatured with $5 \times$ sodium dodecyl sulfate (SDS)-PAGE denatured protein loading buffer (Applygen, Beijing, China) at $98^{\circ} \mathrm{C}$ for $5 \mathrm{~min}$. Next, SDS-polyacrylamide discontinuous gel electrophoresis was used to separate the denatured protein; then, the protein was transferred to nitrocellulose filter membranes (Millipore, Boston, MA, USA) by wetting transfer method for $90 \mathrm{~min}$. Immunoblotting was conducted using specific antibodies listed in Supplementary Table 2 and horseradish peroxidase-conjugated secondary antibody. The membranes were visualized using ChemiDoc XRS+ System (Bio-Rad, Hercules, CA, USA), and the grayscale value of the proteins was estimated using Image Lab 6.0.1.

\section{Immunofluorescence Staining}

Differentiated HSMM myotubes or primary myotubes were fixed in 95\% ethanol. Then, $0.3 \%$ Triton X-100 was used for $15 \mathrm{~min}$ for cell membrane penetration. The samples were washed three times, for 5 minutes each time, and then blocked with goat serum for $2 \mathrm{~h}$. The samples were then incubated with anti-HuR (1:500; Abcam, Cambridge, MA, USA), anti-MyoG (1:250; Abcam), and antimyosin heavy chain (MyHC; 1:400; Santa Cruz Biotechnology, Dallas, TX, USA) overnight at $4^{\circ} \mathrm{C}$, and then with Alexa 488conjugated or Alexa 555-conjugated secondary antibody (Abcam) for $30 \mathrm{~min}$ at $25^{\circ} \mathrm{C}$. Next, $4^{\prime}$,6-diamidino-2-phenylindole (DAPI; Beyotime) was used to stain the cell nuclei, which were observed under a fluorescence microscope (Olympus, Tokyo, Japan). The degree of differentiation was measured by a fusion index, which is defined as the number of nuclei in the myotube and as a percentage of the total nuclei.

\section{Statistical Analysis}

Continuous data were expressed as means \pm standard deviation for normal distribution or as median and interquartile range for those without normal distribution. Student's $t$-test was used for separating the means of the normal data, whereas MannWhitney $U$ test for data that deviated from normal. Spearman's rank-order correlation was used to test correlations of data with non-normal distribution. A $p$ value $<0.05$ was considered as statistically significant. SPSS 22.0 (IBM, Armonk, NY, USA) and GraphPad Prism 8.0 (GraphPad Software, San Diego, CA, USA) were used for all statistical analyses.

\section{RESULTS}

\section{Clinical Characteristics of Patients With IMNM}

The clinical characteristics of the patients with IMNM are presented in Table 1. Of the 18 patients with IMNM, 61.1\% were female. The average disease onset age was 47.6 years, and the median disease duration was 5 months. 12 patients were positive to the anti-SRP-autoantibody, 2 to the anti-HMGCRautoantibody, and the remaining four patients were myositisspecific autoantibody negative. The average VAS score was 4.7, and the average MMT8 score was 58.0.

\section{miR-18a-3p Expression in Skeletal Muscles of Patients With IMNM}

We found that the expression level of miR-18a-3p was significantly higher in patients with IMNM than in the HCs 
TABLE 1 | Clinical features of immune-mediated necrotizing myopathy (IMNM) patients.

\begin{tabular}{lcc}
\hline Characteristics & Patients $(\boldsymbol{n}=\mathbf{1 8})$ & HCs ( $\mathbf{n = 8})$ \\
\hline Female, no. (\%) & $11(61.1 \%)$ & $5(62.5 \%)$ \\
Age of onset, mean \pm s.d. years & $47.6 \pm 15.6$ & $50.8 \pm 17.1$ \\
Disease duration, median of IQR months & $5(2-9.7)$ & \\
Clinical features, no. (\%) & & \\
Muscle weakness & $15(83.3 \%)$ \\
Myalgia & $6(33.3 \%)$ \\
Laboratory data & \\
MSA, no. (\%) & \\
$\quad$ Anti-SRP- positive & $12(66.6 \%)$ \\
$\quad$ Anti-HMGCR- positive & $2(11.1 \%)$ \\
$\quad$ MSA-negative & $4(22.2 \%)$ \\
CK (IU/L), median (IQR) & $3597(1356-7521)$ \\
ALT (IU/L), median (IQR) & $207.5(61.4-319)$ \\
AST (IU/L), median (IQR) & $119.5(39.7-160.8)$ \\
LDH (IU/L), median (IQR) & $674(426.5-1113)$ \\
ESR (mm/h), median (IQR) & $7(5-20.5)$ \\
CRP (mg/dl), median (IQR) & $0.36(0.25-0.45)$ \\
MMT8, mean \pm S.d. & $58.0 \pm 12.9$ \\
PGA VAS, mean \pm S.d. & $4.7 \pm 1.8$ \\
\hline Average valus or numbers & \\
\hline
\end{tabular}

Average values or numbers of each group are shown. Standard deviation (s.d.), interquartile range (IQR), or percentages are shown. MSA, myositis-specific antibody; IMNM, immune-mediated necrotizing myopathy; SRP, signal recognition particle; HMGCR, 3-hydroxy-3-methyl coenzyme A reductase; CK, creatine kinase; ALT, alanine aminotransferase; AST, aspartate aminotransferase; $L D H$, lactate dehydrogenase; ESR, erythrocyte sedimentation rate; CRP, C-reactive protein; PGA, physician global assessment; VAS, visual analog scale; MMT8, manual muscle test 8 (0-80).

$(p=0.0002)$ (Figure 1A). Since miR-18a-3p is the member of miR-17-92 cluster, and the miR-17-92 cluster participates in skeletal muscle differentiation (7), we further detected the expression of other miRNAs in this cluster in these samples (Supplementary Figures 1A-E). The Kyoto Encyclopedia of Genes and Genomes pathway enrichment analysis revealed that the target genes of miR-18a-3p were involved in regulating numerous pathways, ras and mitogen-activated protein kinase (MAPK) signaling pathway are involved in the regulation of myogenic differentiation (21) (Figure 1B). Bioinformatics analysis predicted that $\mathrm{HuR}$ may be a target gene of miR-18a-3p (Figure 1C). Dual-luciferase reporter gene assay showed that overexpression of miR-18a-3p decreased the luciferase activity of the plasmid containing HuR-WT, that is, the luciferase activity of miR-18a-3p binding sequence; however, it did not decrease the activity of HuR-MUT (Figure 1D). Furthermore, overexpression of miR-18a-3p strongly suppressed HuR transcription at $24 \mathrm{~h}$ post-transfection (Figure 1E). Accordingly, western blot analysis showed that the protein levels of $\mathrm{HuR}$ were reduced by the transfection of the miR-18a-3p mimics (Figures 1F, G). These results demonstrated that miR-18a-3p may directly interact with the predicted target site in $\mathrm{HuR}$.

\section{HuR Expression Levels in Patients With IMNM}

Our results showed that the expression level of HuR was significantly reduced in patients with IMNM than in HCs $(\mathrm{p}=0.002)$ (Figure 2A), but the expression of HuR in DM patients is significantly higher than in HCs (Supplementary Figure 2). Western blot analysis revealed a decrease in the protein levels of $\mathrm{HuR}$ in patients with IMNM than in the HCs (Figures 2B, C). At the same time, miR-18a-3p expressed in patients with IMNM were negatively correlated with those of HuR ( $r=-0.512, p=0.029$; Figures 2D). Furthermore, MyoG was highly expressed in patients with IMNM, and its transcription levels were positively correlated with those of $\operatorname{HuR}(r=0.493, p=$ 0.037; Figures 2E, F). Besides, the VAS score of patients with IMNM was positively correlated with HuR levels $(r=0.576, p=$ 0.012); however, the muscle activity component of the Myositis Disease Activity Assessment Tool by VAS (MDAAT-muscle) was negatively correlated with miR-18a-3p expression $(r=$ $-0.550, p=0.017$; Figures 2G, H). No association was identified between HuR or miR-18a-3p expression and the MMT8 score.

\section{Involvement of HuR in Myoblast Differentiation}

As shown in Figures 3A, B, the nuclear abundance of HuR did not change noticeably during differentiation, whereas the cytoplasmatic abundance increased remarkably at the onset of differentiation and remained elevated throughout the process. We observed that the expression levels of MyoG were consistent with those of cytoplasmic HuR; these results were confirmed using immunofluorescence staining of human myoblasts after differentiation (Figure 3C). Next, we use siRNA technology to solve the role of $\mathrm{HuR}$ in myoblast. Three different siRNAs were designed to target human $\mathrm{HuR}$ and transfected in myoblast. The results showed that siHuR effectively down-regulated the mRNA expression in myoblast (Supplementary Figures 3A). According to qRTPCR analysis, transfection of siHuR $(100 \mathrm{nM})$ significantly reduced the cellular level of $\mathrm{HuR}(>70 \%)$ (Supplementary Figures 3B). Besides, we observed that transfection of siHuR significantly inhibited the expression levels of $\mathrm{HuR}$ and $\mathrm{MyoG}$ during differentiation (Figures 3D, E). Western blot analysis showed that the protein levels of $\mathrm{HuR}, \mathrm{MyoG}$, and MyHC in siHuR-treated myoblasts were significantly decreased when compared with that in the control (Figures 3F-I). These results suggested that $\mathrm{HuR}$ knockdown could inhibit myoblast differentiation.

\section{HuR Regulation by miR-18a-3p in Myogenic Differentiation}

As shown in Figure 4A, miR-18a-3p mimics transfection significantly increased miR-18a-3p expression. MyHC immunofluorescence staining on day 5 of differentiation showed that overexpression of $\mathrm{miR}-18 \mathrm{a}-3 \mathrm{p}$ reduced the fusion rate of myoblasts in comparison to that of the mimics NC (Figures 4B, C). Furthermore, miR-18a-3p mimics transfection significantly suppressed the transcription and protein levels of HuR, MyoG, and MyHC (Figures 4D-F). The opposite results 
A

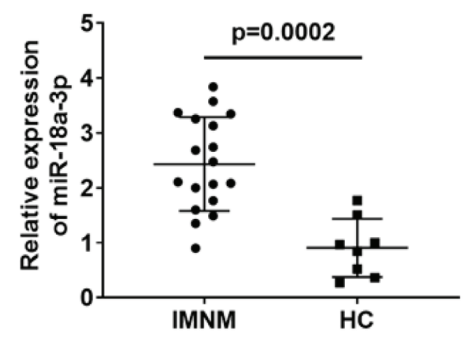

C

5' GCCUUUUCCUGGCCAAGGGCAGc 3' HuR UTR

3' GGUCUUCCUCGUGAAUCCCGUCa 5' miR-18a-3p

5' GCCUUUUCCUGGCCAUUAAGUAc 3'HuR UTR (mutant)

D

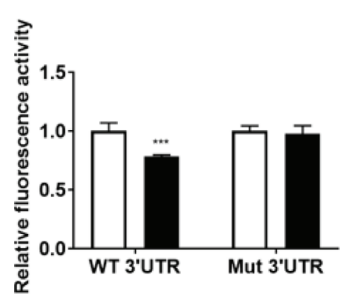

E

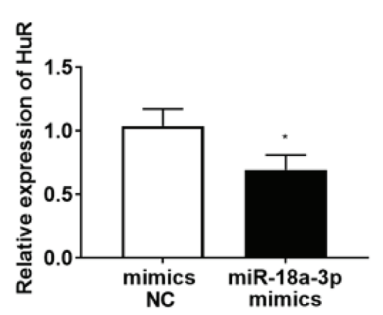

B
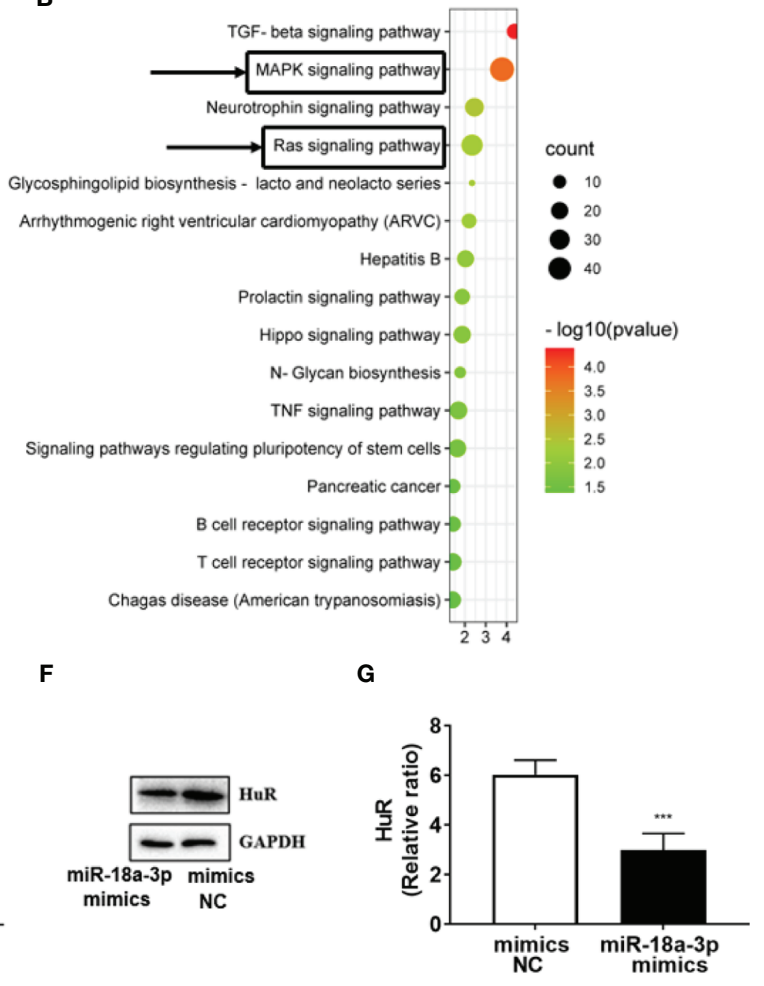

FIGURE 1 | miR-18a-3p is highly expressed in skeletal muscle of patients with immune-mediated necrotizing myopathy (IMNM). (A) miR-18a-3p levels in patients with IMNM and healthy controls (HCs) as determined by quantitative reverse-transcription polymerase chain reaction PCR (qRT-PCR). (B) Kyoto Encyclopedia of Genes and Genomes (KEGG) pathway analysis of miR-18a-3p target genes. (C) Sequence alignment of the 3' untranslated region (UTR) of HuR mRNA with miR-18a-3p. Nucleotides in deletion mutant (Mut) HuR binding sites are marked in red. (D) Effects of miR-18a-3p on the activity of luciferase reporters, bearing wild-type (WT) or Mut on the binding sites of the $3^{\prime}$ UTR of HuR mRNA. (E) HuR levels at $24 \mathrm{~h}$ post-transfection with miR-18a-3p mimics as determined by qRT-PCR. (F) Protein levels of HuR at $24 \mathrm{~h}$ post-transfection with miR-18a-3p mimics as determined by western blot analysis. (G) Glyceraldehyde 3-phosphate dehydrogenase (GAPDH) was used as an internal control for western blot analysis to normalize the relative protein expression. Relative mRNA expression was estimated using the 2- ${ }^{\Delta \Delta \mathrm{Ct}}$ method. Data are expressed as means \pm standard deviation (SD). ${ }^{\star}$, ${ }^{\star \star \star}$ indicate significant differences of $p<0.05$ and $p<0.001$, respectively. miR, microRNA; NC, negative control.

were obtained after transfection of the miR-18a-3p inhibitor (Figures 5A-F). Our data demonstrated that inhibition of miR-18a-3p might promote myogenic differentiation by targeting $\mathrm{HuR}$.

\section{DISCUSSION}

IMNM is a systemic disease characterized by chronic inflammation in muscle tissues, causing weakness and myofiber necrosis (1). In the present study, we explored the role of differentially expressed miRNAs and target genes in regulating the regeneration and differentiation of muscle tissues in patients with IMNM.

miRNA is involved in the regulation of a wide range of developmental and physiological cell processes, including differentiation, proliferation, growth, and apoptosis (22).
Previous studies have shown that miRNAs are abnormally expressed in the muscles of patients with IIM and are probably involved in disease pathogenesis. For instance, miR-221, miR155, miR-146b, miR-214, and miR-222 are upregulated in 10 primary muscle diseases, including IIM (23). Furthermore, miRNAs found in skeletal muscles and myocardium, such as miR-1, miR-133a/b, miR-208, miR-486, miR-206, and miR499 , participate in the myogenic process by modulating the expression of myogenic regulatory factors (24). Of these, the expression of $\mathrm{miR}-1$ and $\mathrm{miR}-133 \mathrm{a} / \mathrm{b}$ is decreased in the muscle tissues of patients with IIM and is negatively correlated with that of TNF- $\alpha$. The latter inhibits the expression of myogenic miRNAs in an NF- $\kappa \mathrm{B}$-dependent manner, suppressing the differentiation of $\mathrm{C} 2 \mathrm{C} 12$ myoblasts into myotubes and leading to muscle regeneration (25). In our study, miR-18a-3p was upregulated in patients with IMNM and participated in myogenic differentiation by regulating HuR expression. We also found that miR-18a-3p expression 


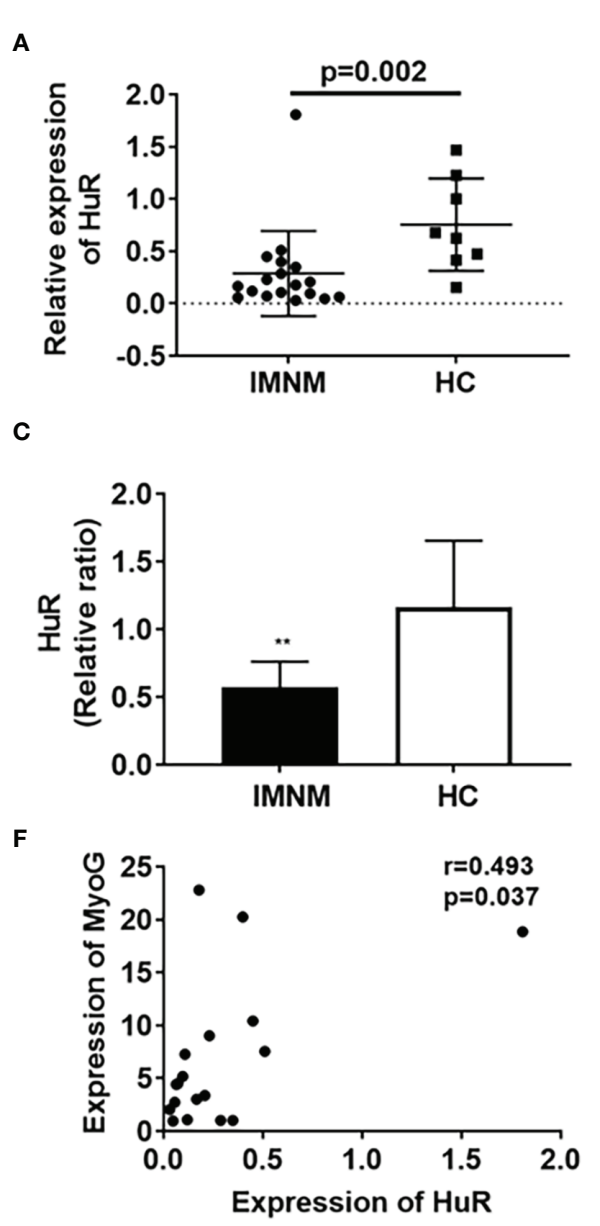

B

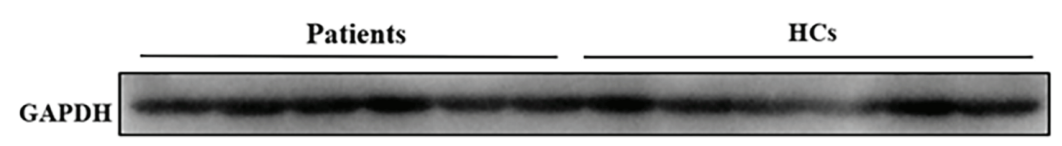

HuR

D
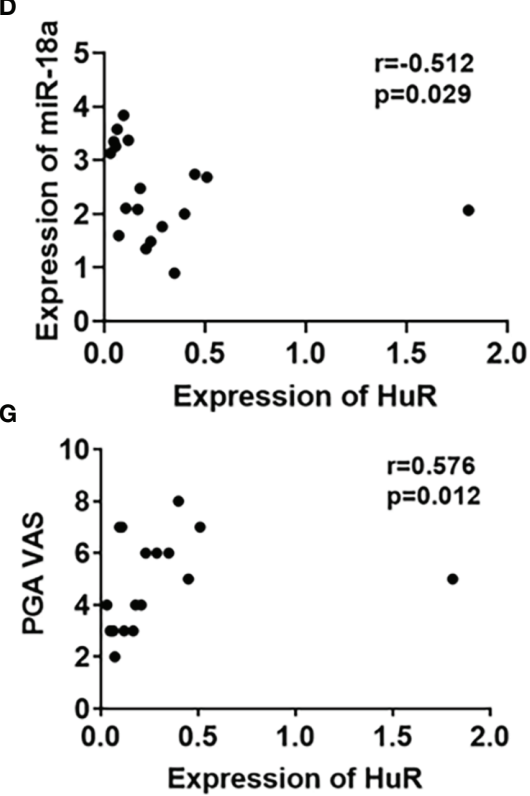

E

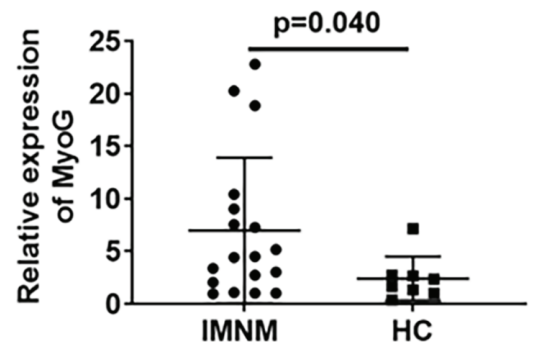

H

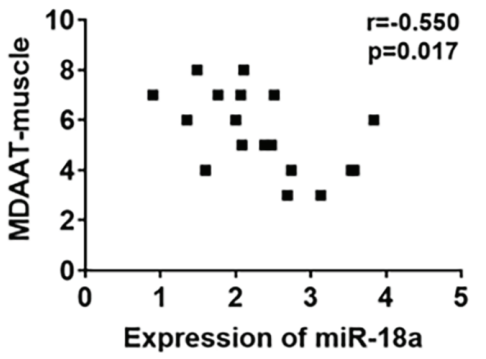

FIGURE 2 | The mRNA and protein levels of HuR are decreased in skeletal muscles of patients with immune-mediated necrotizing myopathy (IMNM). (A) mRNA levels of HuR in patients with IMNM and healthy controls (HCs) as determined by qRT-PCR. (B) Protein levels of HuR in patients with IMNM and HCs as determined by western blot analysis. (C) GAPDH was used as an internal control for western blot analysis to normalize the relative protein expression. (D) Correlation of HuR and miR-18a-3p mRNA levels in patients with IMNM. (E) mRNA levels of myogenin (MyoG) in skeletal muscles of patients with IMNM and HCs as determined by qRTPCR. (F) Correlation of HuR and MyoG mRNA levels in patients with IMNM. (G) Correlation of HuR mRNA levels and Patient Global assessment of disease activity by VAS (0-10). (H) Correlation of miR-18a-3p levels and the muscle activity component of the Myositis Disease Activity Assessment Tool by VAS (MDAAT-muscle). Relative mRNA expression was estimated using the $2^{-\Delta \Lambda C t}$ method. Data are expressed as means \pm standard deviation (SD). ${ }^{* \star}$ indicate significant differences of $p<0.01$.

was negatively related to the VAS score, revealing the potential role of this miRNA in muscle inflammation and the degenerative pathology of IMNM. A previous study on mice with severe myositis has shown that increased expression of inflammatory miRNAs (miR-146a, miR-142-3p, miR-142$5 \mathrm{p}$, and $\mathrm{miR}-455-5 \mathrm{p}$ ) and targeted muscle dystrophins (miR146a, miR-146b, miR-31, and miR-223) are regulated by $\mathrm{NF}-\kappa \mathrm{B}$, and may be related to muscle injury, inflammation, and myasthenia (12). Of note, in our study, we found that the expression of HuR in IMNM was decreased compared to HC, while the expression of $\mathrm{HuR}$ in the muscle tissue of $\mathrm{DM}$ patients was significantly higher than in the HC. The results of this study further indicate that the muscle damage mechanism of DM may be different from that of IMNM.
The miR-17-92 cluster participates in normal development, skeletal myogenesis, immune disease, cardiovascular disease, and tumorigenesis $(26,27)$, of which miR-20a, miR-92, and miR-17 regulate the proliferation of myoblasts but inhibit their differentiation by targeting ENH1. Further, miR-17 and miR20a effectively promote the differentiation of C2C12 cells; whereas miR-18a delays the differentiation of the same cells $(7,28)$. Here, we use bioinformatics software and luciferase reporter gene detection to show that $\mathrm{HuR}$, a RNA-binding protein, is the direct target of miR-18a-3p. Under the overexpression miR-18a-3p, the expression level of HuR RNA and protein was significantly inhibited, and further inhibit myogenic differentiation. On the contrary, interference of miR$18 \mathrm{a}-3 \mathrm{p}$ promoted the expression of $\mathrm{HuR}$ and further promote 
A

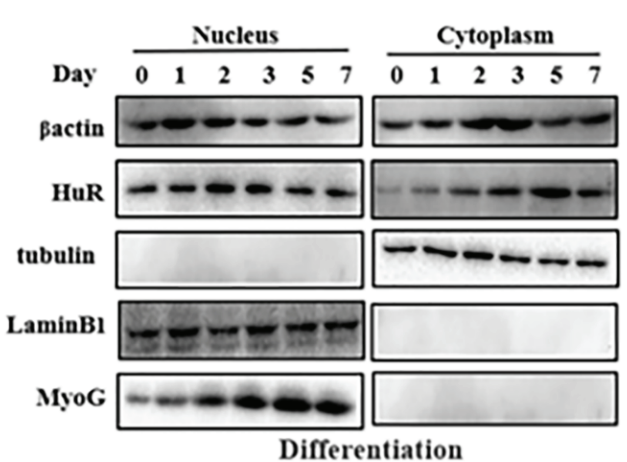

B

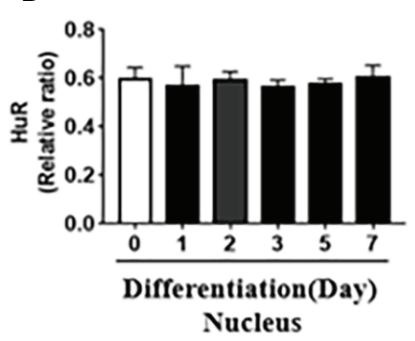

D

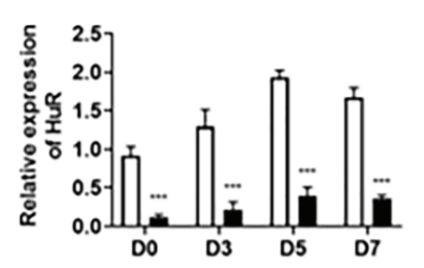

G

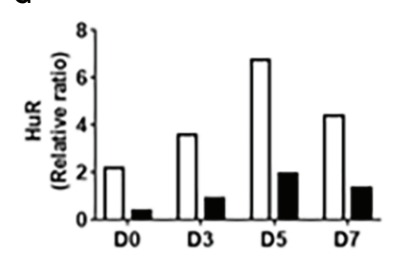

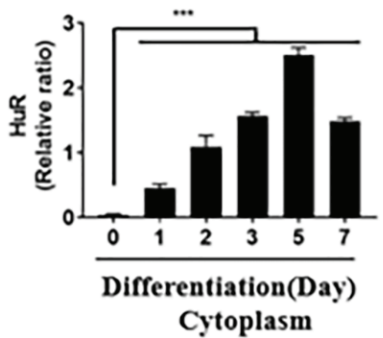

E
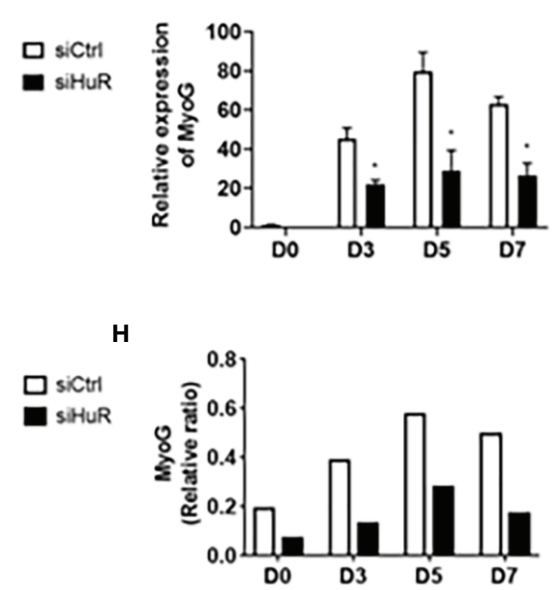

C
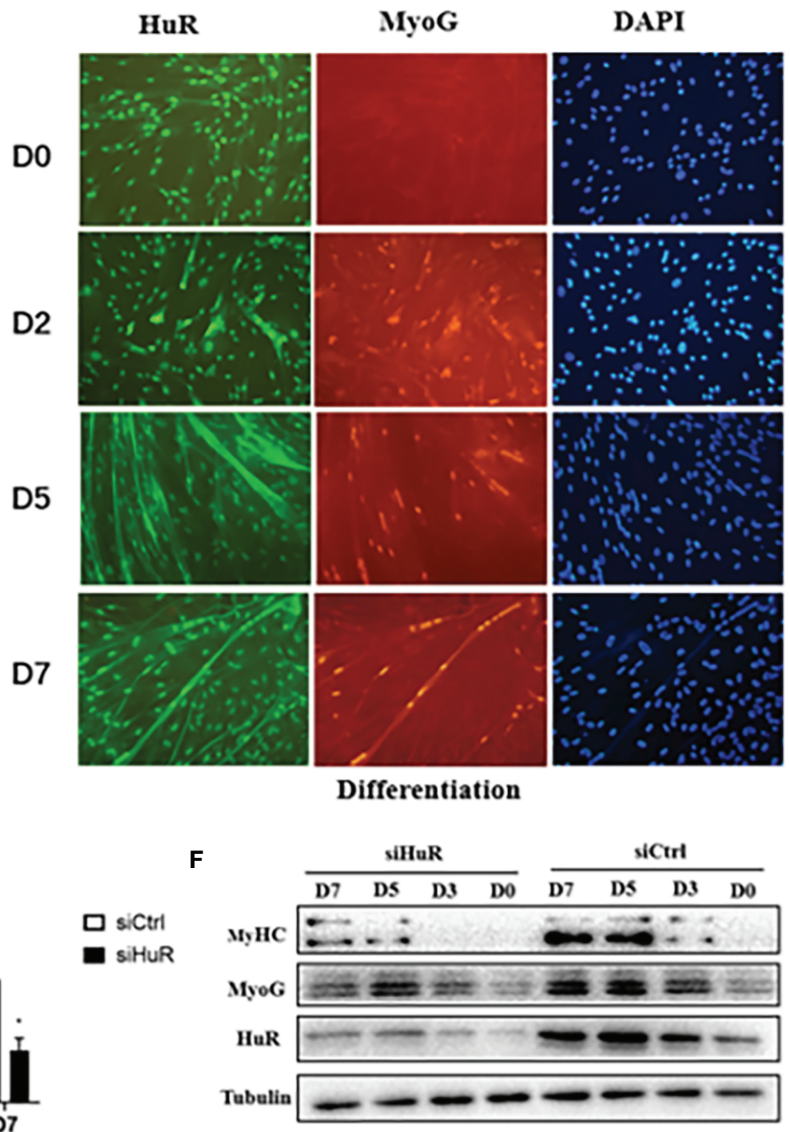

I
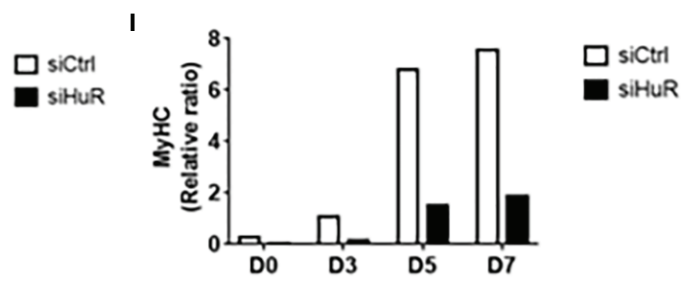

FIGURE 3 | Expression of HuR in human skeletal muscle myoblasts (HSMM) during differentiation. (A) Nuclear and cytoplasmic HuR and myogenin (MyoG) protein levels in HSMM during differentiation as determined by western blot analysis. (B) HuR levels in the human myoblast nucleus as normalized with lamin-B1 (left) and in the human myoblast cytoplasm as normalized with tubulin (right). (C) Immunofluorescence detection of HuR (green) and MyoG (red) in HSMM during differentiation. DAPI (blue) is used as a nuclear stain. Scale bar, $100 \mu \mathrm{m}$. (D) mRNA levels of HuR post-transfection with small interfering RNA targeting HuR (siHuR) at days 0, 3, 5, and 7 of differentiation as determined by qRT-PCR. (E) mRNA levels of MyoG post-transfection with siHuR at days $0,3,5$, and 7 of differentiation as determined by qRT-PCR. (F) Western blot analysis was used to determine HuR, MyoG, and MyHC protein levels post-transfection with siHuR at days $0,3,5$, and 7 of differentiation (G-I) GAPDH was used as an internal control for western blot analysis to normalize relative protein expression. Relative mRNA expression was estimated using the $2^{-\Delta \Delta \mathrm{Ct}}$ method. Data are expressed as means \pm standard deviation (SD). ${ }^{*}{ }^{* \star \star}$ indicate significant differences of $p<0.05$ and $p<0.001$, respectively.

myogenic differentiation. In our study, we found that expression of miR-18a-3p in patients with IMNM was negatively correlated with HuR. miR-18a-3p affected myoblast differentiation by downregulating the expression of HuR. In contrast, use of an miR-18a-3p inhibitor led to opposite results.

$\mathrm{HuR}$ is an RBP that protects the 3'-UTR of mRNA from decay and degradation by stabilizing the adenylate-uridylate- rich elements. HuR is mainly present in the nucleus, where it combines with the target mRNA, to form a complex that moves to the cytoplasm. When $\mathrm{HuR}$ dissociates from the target mRNA, it returns to the nucleus (29). Previous studies have revealed that $\mathrm{HuR}$ regulates various myogenic factors, including MyoG, MyoD, and p21, and that its cytoplasmic accumulation is essential in myogenesis $(16,30)$. In the present 
A

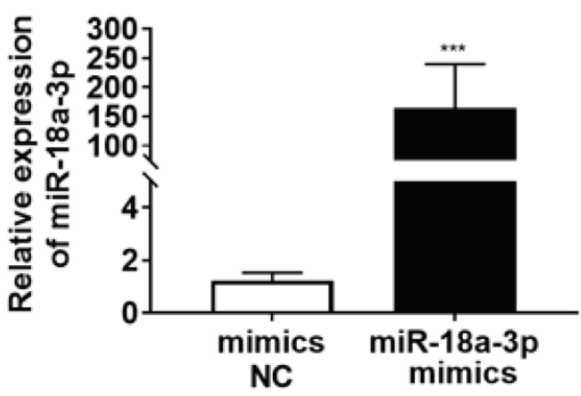

C

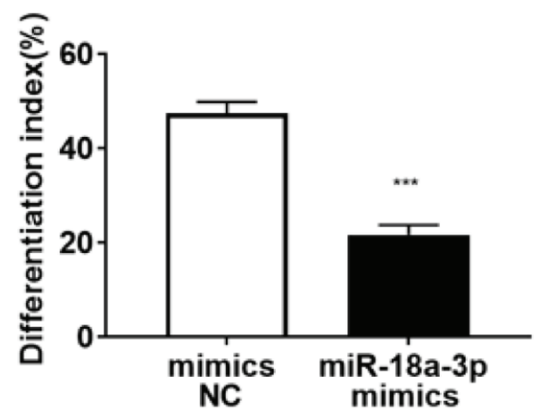

$\mathbf{E}$

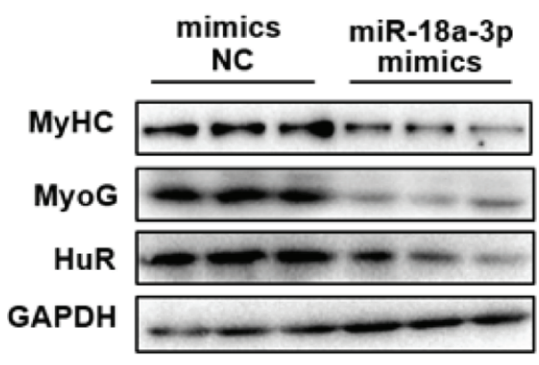

B

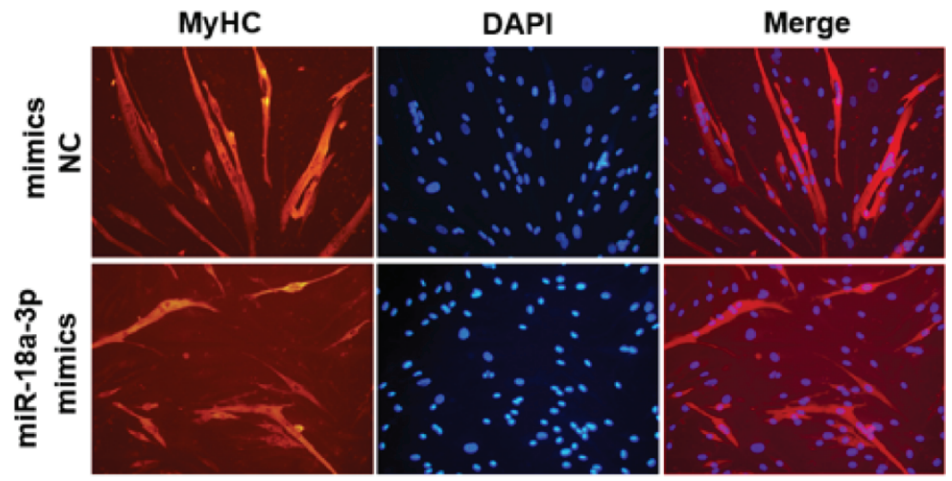

D

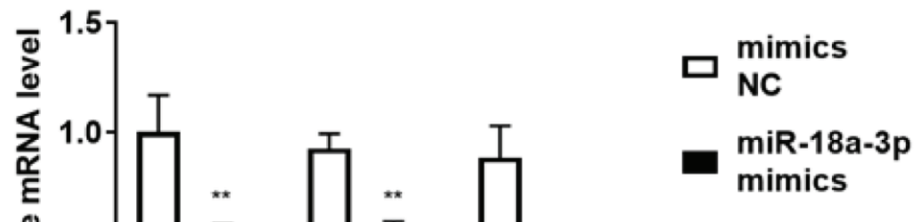

FIGURE 4 | Overexpression of miR-18a-3p inhibits myogenic differentiation. (A) miR-18a-3p levels post-transfection with miR-18a-3p mimics at day 5 of differentiation as determined by qRT-PCR. (B) Immunofluorescence detection of MyHC (red) in human myoblasts. Scale bar, 100 um. (C) Fusion index (ratio of $\mathrm{MyHC}$ positive myotubes with $\geq 2$ nuclei to the total number of nuclei). (D) mRNA levels of HuR, MyoG, and MyHC post-transfection with miR-18a-3p mimics at day 5 of differentiation as determined by qRT-PCR. (E, F) HuR, MyoG, and MyHC protein levels post-transfection with miR-18a-3p mimics at day 5 of differentiation as determined by western blot analysis. Relative mRNA expression was estimated using the $2^{-}{ }^{\Delta \mathrm{Ct}}$ method. Data are expressed as means $\pm \mathrm{standard}$ deviation (SD). ${ }^{*},{ }^{* *},{ }^{* * *}$ indicate significant differences of $\mathrm{p}<0.05, \mathrm{p}<0.01$, and $\mathrm{p}<0.001$, respectively. NC, negative control.

study, we found that the expression of cytoplasmic HuR in myoblasts increased as differentiation progressed and was consistent with that of MyoG. Transfection of siHuR into myoblasts decreased the mRNA and protein levels of MyoG and $\mathrm{MyHC}$ in the differentiated myoblasts, thereby inhibiting cell differentiation.

$\mathrm{HuR}$ is also involved in the pathogenesis of inflammatory diseases (e.g., rheumatoid arthritis) by binding to the mRNAs of inflammatory cytokines (e.g., TNF- $\alpha)(31,32)$. We found that the expression of $\mathrm{HuR}$ was positively correlated with disease activity in patients with IMNM, suggesting that the participation of this protein in inflammatory response is by stabilizing the
mRNAs of inflammatory factors; however, further research is needed to confirm these results.

Overall, our study revealed that miR-18a-3p was significantly upregulated, whereas its target gene $\mathrm{HuR}$ was downregulated in the muscle tissues of patients with IMNM compared to DM and HCs. miR-18a-3p were negatively correlated with muscle activity in patients with IMNM, but the expression levels of HuR were positively correlated with disease activity in patients with IMNM. Finally, miR-18a-3p and HuR might regulate myoblast differentiation (Figure 6). To the best of our knowledge, this is the first study to report the mechanism of miR-18a-3p inhibiting myogenic differentiation in IMNM. 
A

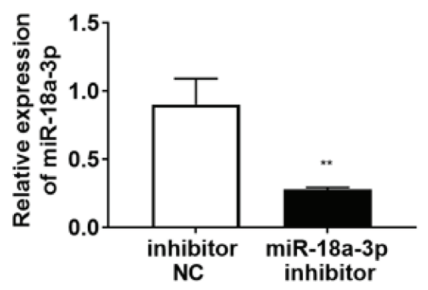

C

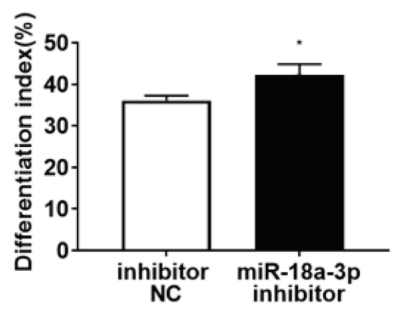

E

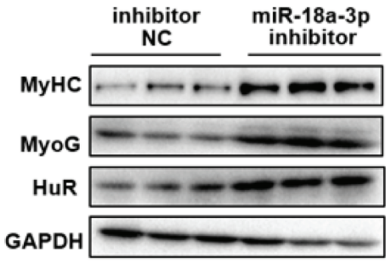

B

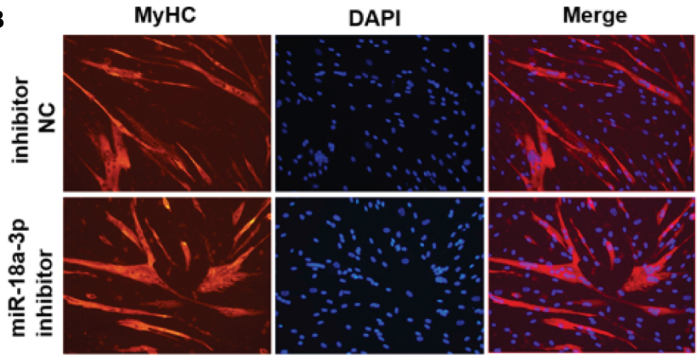

D

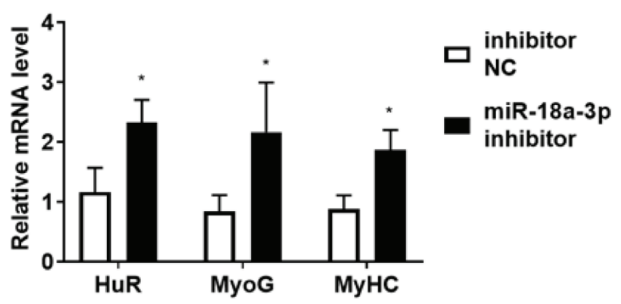

$\mathbf{F}$

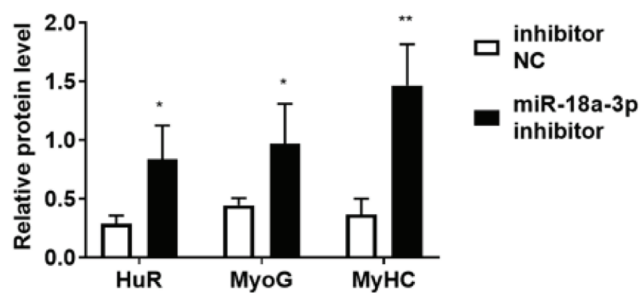

FIGURE 5 | Inhibition of miR-18a-3p promotes myogenic differentiation. (A) miR-18a-3p levels post-transfection with miR-18a-3p inhibitor at day 5 of differentiation as determined by qRT-PCR. (B) Immunofluorescence detection of MyHC (red) in human myoblasts. Scale bar, $100 \mu \mathrm{m}$. (C) Fusion index (ratio of MyHC-positive myotubes with $\geq 2$ nuclei to the total number of nuclei). (D) mRNA levels of HuR, MyoG, and MyHC post-transfection with miR-18a-3p inhibitor at day 5 of differentiation as determined by qRT-PCR. (E, F) HuR, MyoG, and MyHC protein levels post-transfection with miR-18a-3p inhibitor at day 5 of differentiation as determined by western blot analysis. Relative mRNA expression was estimated using the $2-{ }^{\Delta \Delta C t}$ method. Data are expressed as means \pm standard deviation (SD). ${ }^{*},{ }^{* *}$ indicate significant differences of $p<0.05$ and $p<0.01$, respectively. NC, negative control.

A

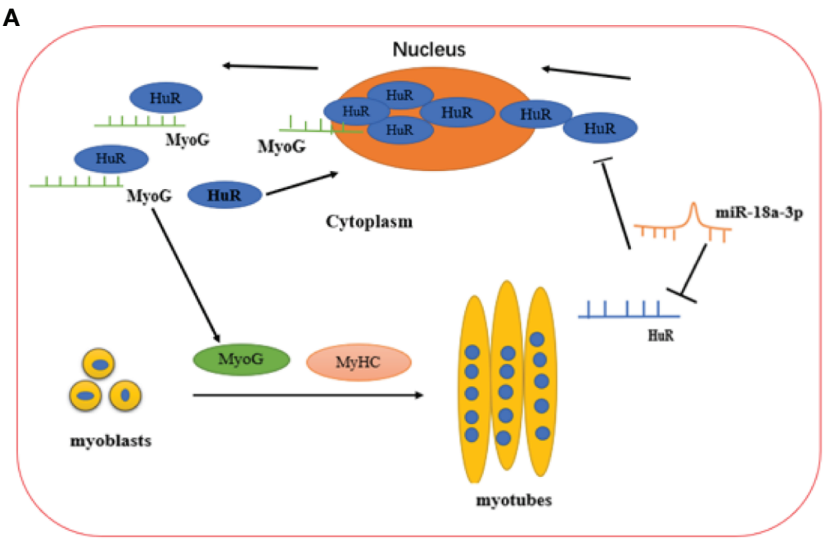

B

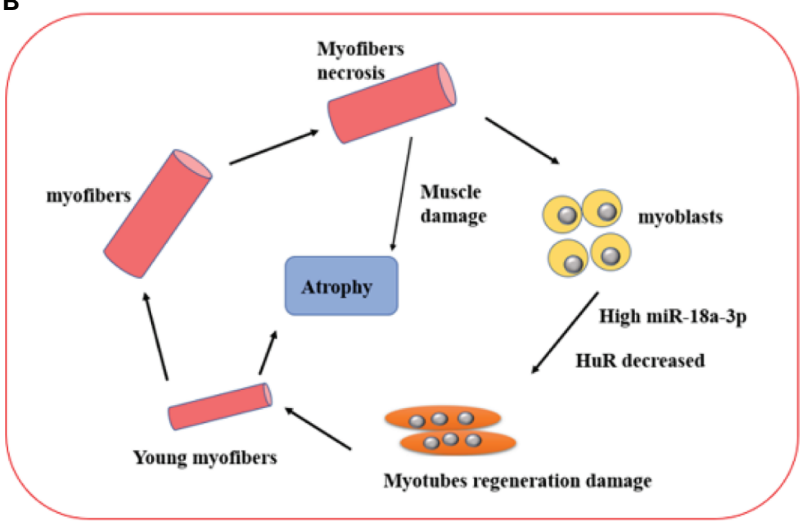

FIGURE 6 | Proposed mechanisms of miR-18a-3p and its target HuR in the IMNM pathogenesis and healthy controls. (A) The physical role of miR-18a-3p and HuR in the myogenesis of skeletal muscle. miR-18a-3p is mainly involved in the post-transcriptional regulation of HuR to inhibit HuR expression. HuR is abundantly localized to the cytoplasm in differentiating myoblasts, which promotes the fusion of myoblasts to form myotubes by stabilizing several myogenic regulatory factor mRNAs such as MyoG. After the myogenesis, HuR returns into the nucleus in the differentiated myotubes. (B) The proposed mechanisms of miR-18a-3p and HuR in the IMNM pathogenesis. Skeletal muscle was damaged and necrosis, which lead to highly expressed miR-18a-3p and decreased HuR, then myoblast regeneration was impaired by the complex interaction of miR-18a-3p and HuR. Ultimately, the skeletal muscle prompt to atrophy. 


\section{DATA AVAILABILITY STATEMENT}

The data presented in the study are available from the corresponding authors upon request.

\section{ETHICS STATEMENT}

The studies involving human participants were reviewed and approved by Ethical Review Committee of the China-Japan Friendship Hospital. The patients/participants provided their written informed consent to participate in this study. Written informed consent was obtained from the individual(s) for the publication of any potentially identifiable images or data included in this article.

\section{AUTHOR CONTRIBUTIONS}

LY, YZ, GW, and XS participated in the conception and design of the experiments. LY and YZ performed the experiments and data analysis. LY, YZ, and XS wrote the manuscript. FC, QP, XL, and GW supervised the manuscript. All authors contributed to the article and approved the submitted version.

\section{REFERENCES}

1. Pinal-Fernandez I, Casal-Dominguez M, Mammen AL. Immune-Mediated Necrotizing Myopathy. Curr Rheumatol Rep (2018) 20(4):21. doi: 10.1007/ s11926-018-0732-6

2. Allenbach Y, Benveniste O, Stenzel W, Boyer O. Immune-Mediated Necrotizing Myopathy: Clinical Features and Pathogenesis. Nat Rev Rheumatol (2020) 16(12):689-701. doi: 10.1038/s41584-020-00515-9

3. Arouche-Delaperche L, Allenbach Y, Amelin D, Mouly V, Mauhin W, Tchoupou GD, et al. Pathogenic Role of Anti-Signal Recognition Protein and Anti-3-Hydroxy-3-Methylglutaryl-CoA Reductase Antibodies in Necrotizing Myopathies: Myofiber Atrophy and Impairment of Muscle Regeneration in Necrotizing Autoimmune Myopathies. Ann Neurol (2017) 81(4):538-48. doi: 10.1002/ana.24902

4. Mok GF, Lozano-Velasco E, Münsterberg A. microRNAs in Skeletal Muscle Development. Semin Cell Dev Biol (2017) 72:67-76. doi: 10.1016/ j.semcdb.2017.10.032

5. Ventura A, Young AG, Winslow MM, Lintault L, Meissner A, Erkeland SJ, et al. Targeted Deletion Reveals Essential and Overlapping Functions of the miR-17 Through 92 Family of miRNA Clusters. Cell (2008) 5):875-86. doi: 10.1016/j.cell.2008.02.019

6. Xiao C, Srinivasan L, Calado DP, Patterson HC, Patterson HC, Zhang B, et al. Lymphoproliferative Disease and Autoimmunity in Mice With Increased miR-17-92 Expression in Lymphocytes. Nat Immunol (2008) 9(4):405-14. doi: 10.1038/ni1575

7. Kong D, He M, Yang L, Zhou R, Yan YQ, Liang Y, et al. MiR-17 and miR-19 Cooperatively Promote Skeletal Muscle Cell Differentiation. Cell Mol Life Sci (2019) 76(24):5041-54. doi: 10.1007/s00018-019-03165-7

8. Sugiyama Y, Yoshimi R, Takeno M, Kunishita Y, Kishimoto D, Kamiyama R, et al. miR-1 Is a Novel Biomarker for Polymyositis/DermatomyositisAssociated Interstitial Lung Disease. Mod Rheumatol (2020) 30(5):878-83. doi: 10.1080/14397595.2019.1661584

9. Gao S, Luo H, Zhang H, Zuo X, Wang L, Zhu H. Using Multi-Omics Methods to Understand Dermatomyositis/Polymyositis. Autoimmun Rev (2017) 16 (10):1044-48. doi: 10.1016/j.autrev.2017.07.021

\section{FUNDING}

This work was supported by the Elite Medical Professionals project of China-Japan Friendship Hospital (NO.ZRJY2021GG14), the Youth Program of the National Natural Science Foundation of China (grant numbers 81401363, 81601367), Major Research Plan of the National Natural Science of China (grant number 91542121), National Natural Science Foundation of China(grant number 81971531),Capital Health Research and Development of Special Programs (grant number 2014-4-4062), and The Fundamental Research Funds for the Central Universities (grant number 3332020074).

\section{ACKNOWLEDGMENTS}

The authors thank the Central Laboratory of China-Japan Friendship Hospital for their assistance in Western blot imaging.

\section{SUPPLEMENTARY MATERIAL}

The Supplementary Material for this article can be found online at: https://www.frontiersin.org/articles/10.3389/fimmu.2021. 780237/full\#supplementary-material

10. Parkes JE, Day PJ, Chinoy H, Lamb JA. The Role of microRNAs in the Idiopathic Inflammatory Myopathies. Curr Opin Rheumatol (2015) 27 (6):608-15. doi: 10.1097/bor.0000000000000225

11. Jiang T, Huang Y, Liu H, Xu Q, Gong Y, Chen Y, et al. Reduced miR-146a Promotes REG3A Expression and Macrophage Migration in Polymyositis and Dermatomyositis. Front Immunol (2020) 11:37. doi: 10.3389/fimmu.2020.00037

12. Kinder TB, Heier CR, Tully CB, van der Muelen JH, Hoffman EP, Nagaraju K, et al. Muscle Weakness in Myositis: MicroRNA-Mediated Dystrophin Reduction in a Myositis Mouse Model and Human Muscle Biopsies. Arthritis Rheumatol (2020) 72(7):1170-83. doi: 10.1002/art.41215

13. Ye L, Zuo Y, Yang H, Li W. Peng Q, Lu X, et al. Specific Autoantibodies and Clinical Phenotypes Correlate With the Aberrant Expression of ImmuneRelated MicroRNAs in Dermatomyositis. J Immunol Res (2019) 2019:2927061. doi: 10.1155/2019/2927061

14. Van der Giessen K, Di-Marco S, Clair E, Gallouzi IE. RNAi-Mediated HuR Depletion Leads to the Inhibition of Muscle Cell Differentiation. J Biol Chem (2003) 278(47):47119-28. doi: 10.1074/jbc.M308889200

15. Von Roretz C, Beauchamp P, Di Marco S, Gallouzi IE. HuR and Myogenesis: Being in the Right Place at the Right Time. Biochim Biophys Acta (2011) 1813 (9):1663-67. doi: 10.1016/j.bbamcr.2011.01.036

16. Figueroa A, Cuadrado A, Fan J, Atasoy U, Muscat GE, Muñoz-Canoves P, et al. Role of HuR in Skeletal Myogenesis Through Coordinate Regulation of Muscle Differentiation Genes. Mol Cell Biol (2003) 23(14):4991-5004. doi: $10.1128 / \mathrm{mcb} .23 .14 .4991-5004.2003$

17. Allenbach Y, Mammen AL, Benveniste O, Stenzel W. 224th ENMC International Workshop: Clinico-Sero-Pathological Classification of Immune-Mediated Necrotizing Myopathies. Zandvoort, The Netherlands, 14-16 October 2016. Neuromuscul Disord (2018) 28(1):87-99. doi: 10.1016/ j.nmd.2017.09.016

18. Rider LG, Feldman BM, Perez MD, Rennebohm RM, Lindsley CB, Zemel LS, et al. Development of Validated Disease Activity and Damage Indices for the Juvenile Idiopathic Inflammatory Myopathies: I. Physician, Parent, and Patient Global Assessments. Juvenile Dermatomyositis Disease Activity Collaborative Study Group. Arthritis Rheum (1997) 40(11):1976-83. doi: 10.1002/art.1780401109 
19. Kim M, Park Y, Kwon Y, Kim Y, Byun J, Jeong MS, et al. MiR-135-5p-P62 Axis Regulates Autophagic Flux, Tumorigenic Potential, and Cellular Interactions Mediated by Extracellular Vesicles During Allergic Inflammation. Front Immunol (2019) 10:738. doi: 10.3389/fimmu.2019.00738

20. Kozomara A, Birgaoanu M, Griffiths-Jones S. Mirbase: From microRNA Sequences to Function. Nucleic Acids Res (2019) 47(1):155-62. doi: 10.1093/ nar/gky1141

21. Bassel-Duby R, Olson EN. Signaling Pathways in Skeletal Muscle Remodeling. Annu Rev Biochem (2006) 75:19-37. doi: 10.1146/annurev.biochem.75. 103004.142622

22. Chen W, Liu D, Li QZ, Zhu H. The Function of ncRNAs in Rheumatic Diseases. Epigenomics (2019) 11(7):821-33. doi: 10.2217/epi-2018-0135

23. Eisenberg I, Eran A, Nishino I, Moggio M, Lamperti C, Amato A, et al. Distinctive Patterns of microRNA Expression in Primary Muscular Disorders. Proc Natl Acad Sci USA (2007) 104(43):17016-21. doi: 10.1073/pnas.0708115104

24. Güller I, Russell AP. MicroRNAs in Skeletal Muscle: Their Role and Regulation in Development, Disease and Function. J Physiol (2010) 588 (pt21):4075-87. doi: 10.1113/jphysiol.2010.194175

25. Georgantas RW, Streicher K, Greenberg SA, Greenlees LM, Zhu W, Brohawn PZ, et al. Inhibition of Myogenic microRNAs 1, 133, and 206 by Inflammatory Cytokines Links Inflammation and Muscle Degeneration in Adult Inflammatory Myopathies. Arthritis Rheumatol (2014) 66(4):1022-33. doi: 10.1002/art.38292

26. Mogilyansky E, Rigoutsos I. The miR-17/92 Cluster: A Comprehensive Update on Its Genomics, Genetics, Functions and Increasingly Important and Numerous Roles in Health and Disease. Cell Death Differ (2013) 20 (12):1603-14. doi: 10.1038/cdd.2013.125

27. Liu QC, Zha XH, Faralli H, Yin H, Louis-Jeune C, Perdiguero E, et al. Comparative Expression Profiling Identifies Differential Roles for Myogenin and P38 $\alpha$ MAPK Signaling in Myogenesis. J Mol Cell Biol (2012) 4(6):386-97. doi: $10.1093 / \mathrm{jmcb} / \mathrm{mjs} 045$

28. Qiu H, Liu N, Luo L, Zhong J, Tang Z, Kang K, et al. MicroRNA-17-92 Regulates Myoblast Proliferation and Differentiation by Targeting the ENH1/
Id1 Signaling Axis. Cell Death Differ (2016) 23(10):1658-69. doi: 10.1038/ cdd. 2016.56

29. Schultz CW, Preet R, Dhir T, Dixon DA, Brody JR. Understanding and Targeting the Disease-Related RNA Binding Protein Human Antigen R (HuR). Wiley Interdiscip Rev RNA (2020) 11(3):e1581. doi: 10.1002/wrna.1581

30. van der Giessen K, Gallouzi IE. Involvement of Transportin 2-Mediated HuR Import in Muscle Cell Differentiation. Mol Biol Cell (2007) 18(7):2619-29. doi: $10.1091 / \mathrm{mbc} . \mathrm{e} 07-02-0167$

31. Suzuki E, Tsutsumi A, Sugihara M, Mamura M, Goto D, Matsumoto I, et al. Expression of TNF-Alpha, Tristetraprolin, T-Cell Intracellular Antigen-1 and $\mathrm{Hu}$ Antigen R Genes in Synovium of Patients With Rheumatoid Arthritis. Int J Mol Med (2006) 18(2):273-8. doi: 10.3892/ijmm.18.2.273

32. Srikantan S, Gorospe M. HuR Function in Disease. Front Biosci (Landmark Ed) (2012) 17:189-205. doi: 10.2741/3921

Conflict of Interest: The authors declare that the research was conducted in the absence of any commercial or financial relationships that could be construed as a potential conflict of interest.

Publisher's Note: All claims expressed in this article are solely those of the authors and do not necessarily represent those of their affiliated organizations, or those of the publisher, the editors and the reviewers. Any product that may be evaluated in this article, or claim that may be made by its manufacturer, is not guaranteed or endorsed by the publisher.

Copyright (c) 2022 Ye, Zuo, Chen, Peng, Lu, Wang and Shu. This is an open-access article distributed under the terms of the Creative Commons Attribution License (CC BY). The use, distribution or reproduction in other forums is permitted, provided the original author(s) and the copyright owner(s) are credited and that the original publication in this journal is cited, in accordance with accepted academic practice. No use, distribution or reproduction is permitted which does not comply with these terms. 